\title{
Commentary
}

\section{Hereditary Hemorrhagic Telangiectasia}

\section{A Model for Blood Vessel Growth and Enlargement}

\author{
Bruce S. Jacobson \\ From the Department of Biochemistry and Molecular Biology, \\ University of Massachusetts, Amberst, Massachusetts
}

Endothelial cell migration, replication, and vessel formation are functions generally considered to make up the initial phase of angiogenesis. Angiogenesis can also be considered to have a major second phase when small vessels enlarge by expansion and recruitment of smooth muscle cells. ${ }^{1,2}$ Angiogenesis, such as occurs in wound repair, or formation of collateral blood vessels following an infarct, ischemia, or reduced blood flow, is certainly advantageous for normal tissue function. One the other hand, angiogenesis in tumor neovascularization, diabetic retinopathy, or vascular malformations that become hemorrhagic or bypass the capillary bed is clearly harmful. Much of the recent work on angiogenesis has focused on the early phase, with less on vessel enlargement. An excellent example of the vessel enlargement phase and a reasonable model with which to study this phase of angiogenesis is the autosomal dominant genetic disorder hereditary hemorrhagic telangiectasia $(\mathrm{HHT})$ or OslerWeber-Rendu disease.

\section{HHT Distribution and Manifestations}

$\mathrm{HHT}$ is widespread among various ethnic groups and ranges in frequency from about 1 in 2000 to 1 in 40,000 depending on the geographical location. ${ }^{3,4}$ The disorder has serious effects, the most common being severe anemia through the loss of blood by hemorrhaging of the telangiectasias in the nose and gut. Larger arteriovenous malformations (AVM), as in the lung (pulmonary AVM or PAVM), occur in more than $20 \%$ of the HHT patients and they percentage could be higher, since they may go undiagnosed. Left-to-right shunting of blood by PAVM can lead to hypoxemia, stroke, and brain abscess. Seventy percent of all PAVM are associated with $\mathrm{HHT} .{ }^{3}$ Brain arteriovenous malformations (cerebral AVM or CAVM) and liver arteriovenous malformations also occur with severe consequences if left unattended. Most CAVM are sporadic, and only about $10 \%$ are HHT-associated. For- tunately, advances in various forms of embolization have led to decreased risk from PAVM and CAVM. Treatment of liver malformations has not been very successful, and the left-to-right shunting of blood may cause heart failure. $\mathrm{HHT}$ is a chronic disease, with telangiectasias and arteriovenous malformations increasing in size and frequency with age. The disease is very diverse and although most serious complications occur later in life, death from the disorder can take place in newborns and juveniles.

\section{Relationship to Angiogenesis}

Interestingly, analysis of $500 \mathrm{HHT}$ patients located through the HHT Foundation International, Inc. suggests the possibility of a significant reduction in the incidence of atherosclerosis and coronary artery disease relative to the general population ( $A E$ Guttmacher, personal communication). It is conceivable that the incidence is actually the same but that it is asymptomatic in HHT patients, possibly due to the increased ability of the patients to form collateral arteries in the heart. This is consistent with HHT being a disease primarily of the vessel enlargement phase of angiogenesis. The role of HHT in the later phase of angiogenesis but not the earlier is also supported by the observation that in the same group of patients studied there was no increased incidence of malignant neoplasias. Vascularization of tumors and onset of malignancy is firmly dependent on the earlier phase of angiogenesis. ${ }^{5}$ This strengthens the view that understanding the molecular and cellular basis of HHT will not only advance our ability to treat those with the disease, but also could serve as a paradigm to uncover the molecular and cellular basis for the blood vessel enlargement aspects of angiogenesis.

Supported in part by National Institutes of Health grant GM 29127 Accepted for publication January 13, 2000

Address reprint requests to Bruce S. Jacobson, Department of Biochemistry and Molecular Biology, University of Massachusetts, Amherst, Massachusetts 01003. E-mail: jacobson@biochem.umass.edu. 
In this issue of The American Journal of Pathology, Bourdeau et al emphasize that although a single mutation in one allele of the endothelial cell surface protein endoglin is essential to induce expression of HHT type 1 (HHT1 ), it is unlikely that the development of arteriovenous malformations is due to a localized reduction of endoglin in the endothelium. ${ }^{6}$ Endoglin was found to be equally present in the arteriovenous malformations and in the phenotypically normal endothelia. This strongly implicates the need for a so-called second hit to initiate the formation of telangiectasias and the larger arteriovenous malformations. The article also describes for the first time a new mutation in the endoglin gene not present in the parents. ${ }^{6}$ Of the 30 families studied to date, all have different endoglin mutations. In this case, if the frequency of one new mutation in 30 were to hold up with a larger sample size, it would be consistent with a greater frequency in the general population than currently thought. This is also supported by the concern that HHT could be underdiagnosed. ${ }^{3,4}$

\section{Endoglin Mutations and Role in HHT}

Evidence that mutations in endoglin are responsible for HHT was demonstrated in $1994,{ }^{7}$ though endoglin was identified almost a decade earlier. ${ }^{8}$ In addition to its involvement in HHT, endoglin is up-regulated during angiogenesis, wound healing, and inflammation. Through a series of elegant experiments ${ }^{9-12}$ it has been shown that endoglin is an accessory protein in the TGF- $\beta$ family of receptor signaling systems. Research into the role of the TGF- $\beta$ family of growth factors and their receptors in general and in angiogenesis is a continuing process, and many reviews on the subject have been published. ${ }^{13-16}$ The TGF- $\beta$ family signaling system is basically composed of two receptors. The type II receptors phosphorylate the type I receptors when cross-linked into a heteromeric complex by growth factors such as TGF- $\beta$, activin, or bone morphogenic protein (BMP). This in turn leads to the phosphorylation of a cytosolic protein Smad by the type I receptor component of the complex. Smads eventually activate transcription in the nucleus through the phosphorylation of other Smads. ${ }^{15}$ Actually, the TGF- $\beta$ signaling complex is most likely a hexomer composed of dimers of both receptors and the growth factor.

Endoglin is a transmembrane homodimeric protein that binds to a number of growth factors in the TGF- $\beta$ superfamily only when the growth factors are bound to their cognate receptors. Endoglin, like betglycan, is considered an accessory protein in the TGF $\beta$ signaling system and does not have any intrinsic signaling capability. Though endoglin and betaglycan share structural similarity, they do not function identically. Endoglin appears to activate TGF- $\beta$ signaling by both facilitating the formation of and becoming part of the type II receptor, type I receptor, TGF- $\beta$ hexomeric complex. ${ }^{12}$ Because endoglin is also a homodimer transmembrane protein, the TGF- $\beta$ signaling complex can be thought of as an octomer with a hexomer of proteins spanning the plasma membrane to propagate signaling from the extracellular growth factors to the cytosolic Smads. ${ }^{12,15}$ It has been postulated that endoglin might also have the ability to recruit other Smads or downstream second messengers into the signaling complex. ${ }^{12}$ Endoglin not only complexes with TGF- $\beta 1$ and TGF- $\beta 3$ when ligated to their receptors, but also interacts with other ligand receptor complexes including activins and BMP. Cell culture work indicates that activins and TGF- $\beta$ s can inhibit endothelial cell growth, whereas BMPs inhibit growth of smooth muscle cells. Because endoglin facilitates signaling by all of the above growth factors, it is reasonable to comprehend that a mutation in endoglin could alter the constraints placed on growth of endothelial cells and smooth muscle cells, thereby stimulating vessel expansion as seen in $\mathrm{HHT} .{ }^{4}$ This is compounded when one considers that endoglin might also facilitate cross-talk with other growth factors such as vascular endothelial growth factor (VEGF), which is known to regulate both the early phase of angiogenesis and the late vessel enlargement phase. ${ }^{2}$ This leads one to suspect that because endoglin plays a central role as an accessory protein in a variety of signaling mechanisms, the homozygous condition would be lethal, which is thought to be the case in humans and demonstrated to be the case in endoglin knockout mice. ${ }^{17}$

\section{Other Mutations and Consequences}

In addition to endoglin mutations which are responsible for type $1 \mathrm{HHT}$, there are at least two other mutations that initiate the formation of the enlarged vascular malformations in HHT. ${ }^{17}$ One, ALK-1, has been cloned and sequenced ${ }^{18}$ and is considered to be responsible for type $2 \mathrm{HHT}$ (HHT-2). A group of patients with HHT that do not map to either ALK-1 or endoglin probably have a different gene defect, which might be responsible for a type $3 \mathrm{HHT}$ (C Shovlin, personal communication). ALK-1 is a TGF- $\beta$ type I receptor. ${ }^{19}$ As endoglin, ALK-1 is also expressed most vigorously in the vascular endothelium compared with other tissues. ${ }^{20,21}$ It has been demonstrated that ALK-1 being a type 1 receptor interacts with growth factor/receptor complexes composed of either TGF- $\beta 1 /$ TGF- $\beta$ type II receptor or activin/activin type II receptor though no downstream signaling occurs. ${ }^{19,22,23}$ However, when a chimeric receptor composed the ALK-1 extracellular domain and the TGF- $\beta$ type 1 receptor cytosolic domain was transfected into cells in vitro, downstream signaling was observed with TGF $\beta-1$, TGF $\beta-3$, and an unknown serum factor. ${ }^{24}$ Interestingly, endoglin was also shown to bind to ALK-1 in a ligand-independent manner. How ALK-1 regulates HHT has yet to be demonstrated; judging from the above, it will most likely turn out to be a very complex process. It is interesting to note that in general, people with HHT-1 (endoglin mutations) seem to have more severe symptoms than those with HHT-2 (ALK-1 mutations), but whether this reflects the findings that endoglin might be involved with more signaling systems than ALK-1 is yet to be demonstrated.

Deletions, insertions, and nonsense and missense mutations in both endoglin and ALK-1 have been demon- 
strated to lead to $\mathrm{HHT}^{4}{ }^{4}$ In all studies to date with human material it has been shown that all endoglin mutations in $\mathrm{HHT}$ result from haploinsufficiency, leading to reduced amounts of endoglin on the cell surface. ${ }^{25,26}$ It was previously found that umbilical vein endothelial cells grown in vitro from newborns and activated monocytes from adults with HHT produced approximately 50\% the amount of endoglin as controls whether they were missense or deletion mutations, consistent with haploinsufficiency. ${ }^{27}$ It has also been shown that null mutations in endoglin do not produce detectable mRNA transcripts. ${ }^{26}$ Haploinsufficiency is further substantiated by the results presented by Bourdeau et al in their article in this issue of the Journal, ${ }^{6}$ where it is shown that the amount of endoglin relative to controls was approximately $50 \%$ in endothelial cells from a PAVM and a CAVM from two patients. HHTpositive relatives of the patients also expressed reduced levels of endoglin to about $50 \%$ of controls when their activated monocytes were evaluated. Though these data indicate that haploinsufficiency is a major player in $\mathrm{HHT}$, other mechanisms should not be ruled out. For example, it is still possible that HHT could result from dimerization of mutated endoglin with the normal or wild-type endoglin produced by the nonmutated allele to act in a dominant negative mechanism (D Marchuk, personal communication). This is based in part on results that show that dominant negative dimers composed of mutated and wild type endoglin appear on the cell surface of COS cells coexpressing missense mutant endoglin and wildtype endoglin. Additional work needs to be done with $\mathrm{HHT}$ patients to confirm a dominant negative mechanism in humans.

\section{Murine and Human HHT}

Thus far, all HHT patients examined have been heterozygous for endoglin or ALK-1 mutations consistent with the homozygous condition being lethal. Furthermore, all homozygous endoglin knockouts in mice are lethal from embryonic days 8.5 to 11.5 , depending on the genetic background. ${ }^{28-30}$ Interestingly, some mice heterozygous for endoglin with either nonsense or null mutations exhibit a type of vascular malformation similar to that observed in humans, but the formation of the lesion depends critically on the strain of mice used. ${ }^{28,29}$ The malformations are most evident in a strain that is prone to vasculopathies and might have lower levels of TGF- $\beta$ compared with the strains of mice not exhibiting the lesions. ${ }^{29,31,32}$ As previously indicated, it is highly likely that the severity and variability of the malformations seen in mice are due to the involvement of other modifier genes. ${ }^{17,28,29}$ The mice are, therefore, an extremely valuable tool to search for modifier genes by cross-breeding with other knockout mice, but whether the modifier genes in mice and humans will turn out to be the same remains to be seen. Knockout mice, in addition to their usefulness in investigating TGF- $\beta$ signaling, are irreplaceable when it comes to screening potential therapeutic treatments for human $\mathrm{HHT}$. For example, would the myriad of antiangiostatic agents now in various phases of testing be efficacious in the treatment of mouse HHT and possibly human HHT? Most would agree that it is premature to give up the research on the human endoglin and ALK-1 natural knockouts but rather the work should proceed concurrently with the anticipation that a more human-like mouse model of HHT can be found. Along the way, better methods will be found to treat humans with the disease.

\section{Where to Now?}

We are at an interesting place in the study of HHT. On one hand, we know that two genes involved in TGF- $\beta$ signaling are responsible for $\mathrm{HHT}-1$ and $\mathrm{HHT}-2$, though involvement of other genes is quite likely. On the other hand, tremendous strides have been made in the diagnosis and management of the disease since the turn of the last century when Osler, Weber, and Rendu reported on its existence. Where to now? The Bourdeau article ${ }^{6}$ points us in an interesting direction. As indicated above, the absence of a localized loss of endoglin in the focal lesions of HHT suggests a second hit to initiate or trigger the formation of the telangiectasias and the large arteriovenous malformations. Such second hits could be other mutations or clonal variations among the endothelial cells or surrounding tissue cells in the telangiectasia. A closer look at the development of the telangiectasia and its structural characteristics should provide some insight into whether HHT involves only the endothelial cells or other cells as well. It should also be emphasized that the propagation of the growth of the lesion most likely involves regulation by tissue cells, because large arteriovenous malformations as seen in tissues such as lungs, brain, and liver are very seldom seen in the dermis and mucosa, where the smaller telangiectasias are most often found.

\section{HHT Structure Is Consistent with a Second Hit}

Unfortunately, relatively little is known about the cellular aspects of telangiectasias and the larger arteriovenous malformations in various tissues of patients with HHT. Based on careful serial sectioning and image reconstruction of dermal telangiectasias of different sizes, the following developmental scenario has been deduced. ${ }^{33}$ The earliest alteration in the vasculature of $\mathrm{HHT}$ is a focally enlarged or dilated postcapillary venule. Larger telangiectasias are formed when the postcapillary venule increases in size, develops disproportionate layers of smooth muscle cells (about twice that for an unaffected similarly sized venule), and connects through capillaries to dilated arterioles. Further enlargement to about $2 \mathrm{~mm}$ results in the loss of the intervening capillaries. No evidence was seen suggesting that the junctions between the endothelial cells were altered in the lesion. Pericytes or smooth muscle cells were not abnormal except that pericytes in pinpoint telangiectasias had more prominent stress fibers. The most distinguishing feature of the earliest dilated venule seen was perivascular leukocytic in- 
filtrates composed mostly of lymphocytes with a few monocytes/macrophages. Such infiltrates were also seen around the arteriovenous connections but not around the normal vasculature of affected patients. Taken together with the Bourdeau article, ${ }^{6}$ this suggests more firmly that although a reduction of endoglin is essential for the formation of telangiectasias, other cells such as smooth muscle cells, lymphocytes. or even dendritic cells might be involved in triggering and/or propagating the formation of telangiectasias. It is important to emphasize that far more research needs to be done on HHT at the cellular level, using some of the innumerable antibodies that are currently available for immunomicroscopy and newer DNA probes for in situ hybridization. Such information will no doubt fuel the engine driving development of testable hypotheses on the role of various vascular cells in regulating the elaboration of the arteriovenous malformations in $\mathrm{HHT}$

The hypothesis that leukocytes or the immune system are involved in $\mathrm{HHT}$ has been further substantiated by comparing the structure of telangiectasias in HHT patients with similar lesions seen in a subset of patients with scleroderma. ${ }^{33}$ The HHT telangiectasias and the scleroderma telangiectasias, when they occur, are similar, but scleroderma telangiectasias are not hemorrhagic. Most important is that both types of telangiectasia have similar perivascular infiltration of predominantly lymphocytes with a few monocytes/macrophages. ${ }^{33}$ Further, the telangiectasias in both scleroderma and HHT seem to occur in response to the enlargement of postcapillary venules and reside in localized regions of the dermis and the mucosa. Much is yet to be done regarding the properties of the infiltrated cells, but, as previously indicated, ${ }^{33}$ the infiltrate is consistent with an immunological event as being a trigger or, at least, being involved in the growth of the telangiectasias.

Whether an immunological event is responsible for the second hit initiating localized development of the HHT arteriovenous malformations is yet to be proven. Other second hit possibilities exist that would account for the observation in the article by Bourdeau et al that the reduction of endoglin seen in the HHT lesions is the same as in normal vascular endothelial cells. Loss of heterozygosity $(\mathrm{LOH})$ in the endoglin gene is one possibility, except that on the surface it is inconsistent with the observation by Bordeaux et al of no difference in endoglin production between lesion and nonlesion endothelial cells. One cannot rule out, however, that a subset of the endothelial cells in the lesion have $\mathrm{LOH}$. Answering this question is not trivial, but it will be testable, given future developments of single-cell reverse transcriptase-polymerase chain reaction. ${ }^{34}$

\section{Other Possible Second Hits}

Clonal variation or lineage diversity either in the endothelial cells or smooth muscle cells could provide the second hit necessary for the localized development of the HHT lesions. Clonal variation and lineage diversity is well established in hemopoiesis, ${ }^{35}$ which includes dendritic cells found in the blood vessel wall. ${ }^{36,37}$ Clonal variation has been suggested for smooth muscle cells ${ }^{38}$ and endothelial cells. ${ }^{39}$ Unfortunately, lineage diversity among these cells has not been demonstrated, in that it is yet to be determined whether one cell leads to another with different and stable gene expression. What has been shown and is consistent with clonal variation is exemplified by endothelial cells. When groups of these cells are removed from different vascular beds and grown in culture under identical conditions, the cells from the different tissues express different genes, as indicated by their protein composition. ${ }^{39}$ In this regard, it is interesting to note that it has been known for some time that the endothelial cells within a vascular element have different properties, ${ }^{40}$ but it has not been demonstrated that this results from clonality or genetically stable differences or is due merely to cells responding to localized changes in environment. Taken together, the above leads one to suspect that during the development of vascular beds, selection of endothelial cells with certain traits could occur at various foci, or that, as with hemopoiesis, endothelial cell diversity could develop by somatic mutation or other epigenetic effects resulting in heritable chromatin alterations. It is also possible that postnatal endothelial cell focal alterations could occur through circulating stem cells recruited or trapped within particular regions of the microvasculature, leading to a second hit for telangiectasia formation. This is supported by the observation that circulating stem cells are recruited to sites of angiogenesis in tumors. ${ }^{41}$ Thus, it will be most interesting to determine whether clonal variation or lineage diversity occurs in endothelial cells or smooth muscle cells and whether this contributes to the focal formation of arteriovenous $\mathrm{HHT}$.

\section{Conclusions}

$\mathrm{HHT}$ is a reasonable paradigm to investigate the regulation of the vessel enlargement phase of angiogenesis. The fact that endoglin deficiency in HHT is not localized to just the arteriovenous malformations as presented in the article by Bourdeau et al begs the question of a second hit to initiate telangiectasia formation. It is possible that such a hit could occur in the endothelial cells themselves or within other cells associated with the vascular malformations. It was suggested from microscopic examination that a second hit or modifier effect was due to an immune response arising from perivascular leukocytes at the site of telangiectasias or from the smooth muscle cells, which are present in disproportionate numbers relative to similarly sized vessels. ${ }^{33}$ Though most emphasis was placed on possible scenarios for second hit to initiate growth, it is possible that other hits could take place during pre- and postnatal development that might compensate for or overcome the haploinsufficiency of reduced production of endoglin. This is suggested from the tremendous diversity of expression of HHT lesions in individuals even within the same family and from the observation that incomplete gene penetrance might not be a rare occurrence in $\mathrm{HHT}^{3,4}$ The existence of 
compensatory or positive modifier genes overcoming the effect of endoglin reduction is a critical point because, though the endothelium is readily accessible to gene therapy by way of the bloodstream, replacement of the endoglin gene could have unforeseen outcomes should compensatory genes be active. Another approach for treatment is drug therapy, not just to reduce the symptoms of the disease that range from, for example, migraine headache or excessive epistaxis and gastrointestinal bleeding to transient ischemic attacks, ${ }^{3,4}$ but also to prevent or reverse the growth of the arteriovenous malformations. Discovery of pharmacological treatments will be markedly enhanced as we learn more about the disorder at the biochemical and cellular levels. For example, the TGF- $\beta$ family signaling system is very complex. ${ }^{13-16}$ An advantage of the complexity is that there is likely to be tissue specificity, as indicated by the prevalence of endoglin and ALK-1 in the vascular endothelium, creating targets for regulating TGF- $\beta$ signaling in particular cells or for fine-tuning the signals sent. The speed with which we can devise treatment will be significantly enhanced by paralleling work with the mouse knockouts and more in-depth biochemical and cellular analyses of human tissues, whether they be umbilical cord cells from newborns shown to have HHT or adult patient biopsy material. Fortunately, the HHT Foundation International, through its extensive network of patients, has clearly helped to move the science forward, not only for understanding their own disorder but also to provide better insight into other diseases involving the blood vessel enlargement phase of angiogenesis.

\section{Acknowledgments}

My deepest gratitude is extended to the members of the HHT Foundation International (New Haven, CT, www.hht.org) for their generosity and for providing what has become key to much genetics-based research. I am indebted to Drs. Irwin Braverman, Alan Guttmacher, Michelle Letarte, Douglas Marchuk, Claire Shovlin, Stephen Schwartz, and Robert White for their helpful discussions and reality checks on a variety of ideas.

\section{References}

1. Folkman J, D'Amore P: Blood vessel formation: what is its molecular basis? Cell 1996, 1153-1155

2. Petterson A, Nagy JA, Brown LF, Sundberg C, Morgan E, Jungles S, Carter R, Krieger JE, Manseau EJ, Bliss VS, Eckelhoefer IA, Feng D, Dvorak AM, Mulligan RC, Dvorak HF: Heterogeneity of the angiogenic response induced in different normal adult tissues by VPFR/VEGF. Lab Invest 2000, in press

3. Guttmacher AE, Marchuk DA, White RI: Hereditary hemorrhagic telangiectasia. N Engl J Med 1995, 333:918-924

4. Sholvin CL, Letarte M: Hereditary hemorrhagic telangiectasia and pulmonary artiovenous malformations: issues in clinical management and review of pathogenic mechanisms. Thorax 54:714-729

5. Bicknell R, Lewis CE, Ferrara N. Tumor Angiogenesis. New York, Oxford University Press, 1997, pp 1-381

6. Bourdeau A, Cymerman U, Paquet M, Meschino W, McKinnon WC, Guttmacher AE, Becker L, Letarte M: Endoglin expression is reduced in normal vessels but still detectable in arteriovenous malformation of patients with hereditary hemorrhagic telangiectasia type 1. Am J Pathol 2000, 156:911-923

7. McAllister KA, Grogg KM, Johnson DW, Gallione CJ, Baldwin MA Jackson CE, Helmbod EA, Markel DS, McKinnon WC, Murrell J, et al.: Endoglin, a TGF-beta binding protein of endothelial cells, is the gene for hereditary hemorrhagic telangiectasia type 1. Nat Genet 1994, 8:345-351

8. Quakenbush EJ, Letarte M: Identification of several cell surface proteins of non-T, non-B acute lymphoblastic leukemia by using monoclonal antibodies. J Immunol 1985, 134:1276-1285

9. Gougos A, Letarte M: Primary structure of endoglin, an RGD-containing glycoprotien of human endothelial cells. J Biol Chem 1990, 265: 8361-8364

10. St. Jacques S, Cymerman U, Pece N, Letarte M: Molecular characterization and in situ localization of murine endoglin reveal that it is a transforming growth factor-beta binding protein of endothelial and stromal cells. Endocrinology 1994, 134:2645-2657

11. Yamashita $H$, Ichijo $H$, Grimsby S. Moren A, ten Dijke P, Miyazono K: Endoglin forms a heteromeric complex with the signaling receptors for transforming growth factor-beta. J Biol Chem 1994, 269:19952001

12. Pece-Barbara N, Wrana J, Letarte M: Endoglin is an accessory protein that interacts with the signaling receptor complex of multiple members of the transforming growth factor-beta superfamily. J Biol Chem 1999, 274:584-594

13. Madri J, Sankar S: The biphasic effects of transforming growth factors $\beta$ in angiogenesis. Tumor Angiogenesis. Edited by Bicknell R, Lewis CE, Ferrara N. Oxford, Oxford University Press, 1997, pp 239-249

14. Pintavorn P, Ballermann BJ: TGF-beta and the endothelium during immune injury. Kidney Int 1997, 51:1401-1412

15. Massague J: TGF-beta signal transduction. Ann Rev Biochem 1998, 67:753-791

16. Pepper MS: Transforming growth factor-beta: vasculogenesis, angiogenesis and vessel wall integrity. Cytokine Growth Factor Rev 1997, 8:21-43

17. Sholvin CL: Supermodels and disease: insights from the HHT mice. J Clin Invest 1999, 104:1335-1336

18. Johnson DW, Berg JN, Gallione CJ, McAllister KA, Warner JP, Helmbold EA, Markel DS, Jackson CE, Porteous ME, Marchuk DA: A second locus for hereditary hemorrhagic telangiectasia maps to chromosome 12. Genome Res 1995, 5:21-28

19. Attisano L, Carcamo J, Ventura F, Weis FM, Massague J, Wrana JL: Identification of human activin and TGF beta type I receptors that form heteromeric kinase complexes with type II receptors. Cell 1993, 75:671-680

20. Roelen BA, van Rooijen MA, Mumery CL: Expression of ALK-1, a type 1 serine/threonine kinase receptor, conincides with sites of vasculogenesis and angiogenesis in early mouse development. Dev Dyn 1997, 209:418-430

21. Pancheko MP, Williams MC, Brody JS, Yu Q: Type I receptor serinethreonine kinase preferentially expressed in pulmonary blood vessels. Am J Physiol 1996, 270:L547-558

22. ten Dijke $P$, Yamashita $H$, Ichijo H, Franzen $P$, Laiho M, Miyazano K Heldin $\mathrm{CH}$ : Characterization of type I receptors for transforming growth factor-beta and activin. Science 1994, 264:101-104

23. De Winter JP, De Vries CJ, Van Achterberg TA, Ameerun RF, Feijen A, Sugino $H$, De Waele P, Huylebroeck D, Verschueren K, Van Den Eijden-Van Raaij AJ: Truncated activin type II receptors inhibit bioactivity by the formation of heteromeric complexes with activin type I receptors. Exp Cell Res 1996, 224:323-334

24. Lux A, Attisano L, Marchuk D: Assignment of transforming growth factor beta-1 and beta-3 and a third new ligand to the type I receptor ALK-1. J Biol Chem 1999, 274:9984-9992

25. Pece N, Vera S, Cymerman U, White R, Wrana J, Letarte M: Mutant endoglin in hereditary hemorrhagic telangiectasia type 1 is transiently expressed intracellularly and is not a dominant negative. J Clin Invest 1997, 100:2568-2579

26. Pece-Barbara N, Cymerman U, Vera S, Marchuk D, Letarte M: Expression analysis of four endoglin missense mutations suggests that haploinsufficiency is the predominant mechanism for hereditary hemorrhagic type 1. Hum Mol Genet 1999, 8:1271-1281

27. Cymerman U, Vera S, Pece-Barbara N, Bourdeau A, White RI, Dunn JJ, Letarte M: Identification of hereditary hemorrhagic telangiectasia 
type 1 in newborns by protein expressions and mutation analysis of endoglin. Pediatr Res, 2000, 47:24-35

28. Bourdeau A, Dumont D, Letarte M: A murine model of hereditary hemorrhagic telangiectasia. J Clin Invest 1999, 104:1343-1351

29. Arthur H, Ure J, Smith A, Renforth G, Wilson D, Torsney E, Charlton R, Parums D, Jowett T, Marchuk D, Diamond A: Endoglin, an ancillary TGF receptor, is required for extra-embryonic angiogenesis, and plays a key role in heart development. Dev Biol 2000, 217:42-53

30. Li D, Sorenson L, Brooke B, Urness L, Davis E, Taylor D, Boak B, Wendel D: Defective angiogenesis in mice lacking endoglin. Sci 1999, 284:1534-1537

31. Elsaghier AA, McLaren DJ: Schistosoma mansoni: evidence that vascular abnormalities correlate with the 'non-permissive' trait in 129/ Ola mice. Parasitology 1989, 3:377-381

32. Coulson PS, Wilson RA: Portal shunting and resistance to Schistosoma mansoni in 129 strain mice. Parasitology 1989, 3:383-389

33. Braverman IM, Key A, Jacobson BS: Ultrastructure and three dimensional organization of the telegiectases of hereditary hemorrhagic telengiectasia. J Invest Dermatol 1990, 95:422-427

34. Cheng T, Shen H, Giokas D, Gere J, Tenen D, Scadden D: Tepmoral mapping of gene expression levels during the differentiation of indi- vidual primary hematopoietic cells. Proc Natl Acad Sci USA 93: 13158-13163

35. Enver T, Greaves M: Loops, lineage, and leukemia. Cell 1998, 94 9-12

36. Santiago-Schwartz F: Positive and negative regulation of the myeloid dendritic cell lineage. J Leukoc Biol 1999, 66:209-216

37. Bobryshev YV, Lord RS: Mapping of vascular dendritic cells in atherosclerotic arteries suggests their involvement in local immune-inflammatory reactions. Cardiovasc Res 1998, 37:799-810

38. Schwartz S: The intima: a new soil. Circ Res 1999, 85:877-879

39. Stolz D, Jacobson BS: Macro-and microvascular endothelial cells in vitro: maintenance of biochemical heterogeneity despite loss of ultrastructural characteristics. In Vitro Cell Dev Biol 1991, 27A:169-182

40. Simionescu N, Simionescu M: The cardiovascular system. Cell and Tissue Biology: A Textbook of Histology. Edited by Weiss L. Baltimore, Urban \& Schwarzenberg, 1988, pp 355-400

41. Takayuki A, Masuda H, Takahasi T, Kalka C, Pastore C, Silver M, Kearne M, Isner JM: Bone marrow origin of endothelial progenitor cells responsible for postnatal vasculogenesis in physiological and pathological neovascularization. Circ Res 1999, 85:221-228 